\title{
Why do patients bypass the nearest hospital? An empirical analysis for orthopaedic care and neurosurgery in the Netherlands
}

\author{
Marco Varkevisser · Stéphanie A. van der Geest
}

Received: 14 July 2006/ Accepted: 15 December 2006/ Published online: 26 January 2007

(C) Springer-Verlag 2007

\begin{abstract}
Using data for 2003, we find that both for non-emergency orthopaedic care $(38 \%)$ and neurosurgery (54\%) numerous Dutch patients did not visit the nearest hospital. Our estimation results show that extra travel time negatively influences the probability of hospital bypassing. Good waiting time performance by the nearest hospital also significantly decreases the likelihood of a bypass decision. Patients seem to place a lower negative value on extra travel time for orthopaedic care than for neurosurgery. The valuation of shorter waiting time also varies between these two types of hospital care. A good performance of the nearest hospital on waiting time decreases the likelihood of a bypass decision most for neurosurgery. In both samples, patients are more likely to bypass the nearest hospital when it is a university medical centre or a tertiary teaching hospital. Patient attributes, such as age and social status, are also found to significantly affect hospital bypassing. From our analysis it follows that both patient and hospital care heterogeneity should be taken into account when assessing the substitutability of hospitals.
\end{abstract}

\footnotetext{
M. Varkevisser $(\square)$

Institute of Health Policy and Management (iBMG) and Erasmus Competition and Regulation Institute (ECRi), Erasmus University Rotterdam, Room L3-086, P.O. Box 1738, 3000 DR Rotterdam, The Netherlands e-mail: varkevisser@bmg.eur.nl

S. A. van der Geest

Erasmus Competition and Regulation Institute (ECRi),

Erasmus University Rotterdam, Rotterdam,

The Netherlands
}

Keywords Patient choice behaviour . Hospital bypassing · Hospital competition

JEL Classification $\mathrm{I} 11 \cdot \mathrm{C} 25 \cdot \mathrm{D} 12$

\section{Introduction}

Patients' decisions regarding which hospital to visit have been debated in the health economics literature for several decades. Empirical studies were aimed initially at health planners and hospital administrators, since effective planning and management of health care require models that explain and predict regional hospital utilisation [6]. In the late 1980s, however, a wider range of people became interested in which factors influence patient choice among hospitals. In particular, health insurers who are marketing health plans with a limited set of providers need to know the attributes that affect the choice of hospital. Since many developed countries are experiencing the urgency of incentive-based health system reform, and have started to deregulate hospital markets [3], such knowledge is becoming increasingly important. The international health policy virus of 'reform' also affects Europe; health insurers and patients now have greater freedom of choice in many European Union member states [7]. In the Netherlands, competing health insurers are now allowed to contract selectively. Since 1 February 2005 they have to negotiate contracts with individual hospitals for almost $10 \%$ of total hospital care [11].

Research on patient decision behaviour is especially important for health insurers in deregulated hospital markets. Experience from the United States shows that insurers' bargaining clout depends crucially on their 
ability to channel patients to hospitals with which favourable discounts have been negotiated [13]. For patients, such channelling may imply that they have to bypass the nearest hospital and travel to a more distant hospital. As patients generally dislike travelling, it can reasonably be assumed they would be willing to bypass the nearest hospital only for particular reasons. For example, higher quality of care or shorter waiting times may compensate patients for the inconveniences of increased travel time [8].

This paper is the first empirical analysis of actual hospital visits in the Netherlands (revealed preferences). Using individual patient level non-emergency hospital utilisation data for the year 2003, we estimate a logit model to assess which patient and hospital attributes affected decisions to visit or bypass the nearest hospital. To take the heterogeneity of hospital care into account, we analysed two different medical specialties: orthopaedic care, reflecting a 'regular' type of hospital care, and the more sophisticated medical specialty of neurosurgery. Differences in medical complexity between these two types of care can be illustrated by the percentage of total hospital visits that ultimately result in an inpatient hospital admission. In the Netherlands each year over 515,000 patients need specialised orthopaedic care, of which only around $15 \%$ are admitted as inpatient. For neurosurgery, the corresponding figures are approximately 30,000 and almost $40 \%$, respectively. Our analysis indicates that, for both medical specialties, travel time and hospital attributes as well as patient attributes significantly affect patients' decisions to bypass the nearest hospital.

\section{Empirical literature on revealed hospital bypassing: United States only}

Several previous papers have examined patients' decisions to visit or bypass the nearest hospital. ${ }^{1}$ These papers exclusively analysed hospital bypassing in the United States. Bronstein and Morrisey [1] find that for rural pregnant women in the state of Alabama, travel distances and hospital equipment (reflecting perceived quality differences) were important considerations in the choice of an obstetrics hospital. These authors conclude that rural women with more resources travelled away from their nearest hospital towards hospitals in metropolitan areas, hospitals with high birth volumes, and those with so-called high-risk bassinets.

\footnotetext{
${ }^{1}$ Since this paper focuses on hospital bypassing in particular, we do not discuss the extensive literature on patient hospital choice in general.
}

White and Morrisey [16] report that, in California, bypass rates were higher for more complex procedures (such as back, joint and vascular surgery), and highest for highly complex procedures such as open heart surgery and kidney transplant. They do not, however, control for individual service offerings by hospitals that may bias their results. Tai et al. [14] analyse the hospital bypassing behaviour of rural Medicare beneficiaries. The results of their estimation reveal that distance, hospital attributes (greater size and scope) as well as patient attributes (age and income) had a substantial influence on the decision to visit or bypass the nearest hospital.

\section{Studies on patient behaviour in the Netherlands: stated preferences only}

Prior to the introduction of the new Health Insurance Act in January 2006, Dutch citizens were either enrolled in compulsory social health insurance (about two-thirds of the population) or voluntarily insured with private health insurers (nearly the remaining onethird of the population). Within both health insurance schemes, patients were free to choose any hospital. In the social health insurance scheme, patients' hospital costs were always fully reimbursed; cost sharing arrangements were common only in the private health insurance scheme. Research by ECORYS-NEI [4] indicates that travel time is the most important hospital attribute for Dutch patients, followed by (perceived) hospital expertise and own previous experiences with a hospital. Van der Schee et al. [10] concluded that the Dutch patient's ideal hospital has a good reputation, requires $15 \mathrm{~min}$ of travel time at most, has an $7 \times 24$ emergency department, guarantees each patient a regular physician, has sufficient parking facilities, participates in a regional network of health providers, and has short waiting times. Non-emergency care patients especially seem to prefer hospitals that have a good reputation, while the other attributes (including travel time) are of minor importance to these patients. From NMa [9], it follows that patients in the Netherlands attach a higher value to quality indicators such as reputation than they do to travel time. This result suggests that when hospitals improve quality, patients are willing to accept more travel time.

\section{Conceptual model and estimation method}

In this paper we empirically analyse hospital bypass decisions made by Dutch patients. The empirical 
specification and the underlying conceptual model are similar to those used in previous empirical studies [1, 14]. Both are based on standard utility theory. From this theory it follows that the decision to visit or bypass the nearest hospital is determined by the characteristics of that hospital in combination with specific characteristics of the patient. Theoretically, patients are expected to choose between the nearest hospital and a hospital further away, taking extra travel time and (perceived) quality into consideration. The type of hospital competition that results can be considered as a variant of the standard Hotelling model $[2,15]$.

From previous research it follows that, in the Netherlands, the decision of which hospital to visit is most often made by patients themselves, alone or in consultation with their general practitioner [4]. Since Dutch GPs do not face economic incentives to refer patients to particular hospitals, it is not in their interest to neglect patients' interests when deciding which hospital they should visit. Our empirical specification therefore asserts that patients (or GPs as their agents), given their needs and preferences, decide to visit or bypass the nearest hospital on the basis of its attractiveness. We estimate the following linear logit specification:

$B_{i}=\alpha+\beta T_{i}+\delta X_{i}+\lambda Z_{i}+\varepsilon_{i}$

where $B_{i}$ is a dummy variable that has value 1 when patient $i$ bypassed the nearest hospital providing the medical specialty analysed and value 0 otherwise; $T_{i}$ reflects extra travel time that is required for patient $i$ to reach the next-nearest hospital providing the medical specialty analysed; $X_{i}$ is a vector of patient attributes; $Z_{i}$ is a vector of attributes of the nearest hospital providing the medical specialty analysed; and $\varepsilon_{i}$ is the error term.

We expect the likelihood of a bypass decision to decrease when extra travel time to the next-nearest hospital increases. In addition to this variable, we distinguish nine patient characteristics: gender, age, retirement, unemployment, disability, social security, self-employment, and geographic environment. Note that, since all patients are enrolled in social health insurance, their annual income in 2003 did not exceed $€ 31,750$. Because the opportunity costs of increased travel time are likely to depend on income, the potential impact of any remaining differences in income is expected to be captured by the explanatory variables reflecting the patient's social status (retirement, unemployment, disability, social security, and selfemployment). The effect of gender on patients' bypass decisions is unclear in advance. Older or disabled patients are likely to be less mobile than younger ones and thus less likely to bypass the nearest hospital. Unemployed patients may be more likely to bypass the nearest hospital because their opportunity costs of increased travel time are lower, whereas the opposite may hold for self-employed patients. We expect patients living in urban areas to be more likely to bypass the nearest hospital, because they most often have more nearby hospital alternatives than patients who live in rural areas.

Our specification suggests that patients, given their personal characteristics, will accept the inconvenience and higher costs of travelling to a more distant hospital when they perceive the nearest hospital as unattractive. Based on the insights gained from previous research on stated preferences [10], we examine five important features of the nearest hospital to test their ability to attract patients: university medical centre, tertiary teaching hospital, total number of beds, volume of relevant first hospital outpatient visits, and waiting time performance. Note that, because all patients in our sample are fully insured for hospital care and co-payments are absent, hospital prices do not affect patients' bypass decisions. Due to perceived quality differences, patients may prefer both academic and teaching hospitals over general hospitals. We expect the likelihood of a bypass decision to be negatively affected by hospital size. Patients may prefer larger hospitals that also treat many patients similar to themselves. Relatively low waiting time is also expected to increase the attractiveness of the nearest hospital.

\section{Data}

Our principal data source is the Agis Health Database. ${ }^{2}$ This database contains detailed information on non-emergency first hospital outpatient visits (so-called 'eerste polikliniek bezoeken'; i.e. EPBs) by socially insured Agis enrolees during the year 2003. The available data include patient's age, gender, zip code, social status, and administration number, the medical specialty attended, and the zip code and name of the hospital visited. We extracted observations on hospital visits for orthopaedic services $(n=62,213)$ and neurosurgical services $(n=5,648)$. From these samples we omitted all observations on patients younger than 18 years, because for under-aged children the decision to bypass the nearest hospital may be complicated by

\footnotetext{
${ }^{2}$ In 2003, Agis was one of the largest Dutch health insurers, representing approximately 1.7 million customers of which more than $85 \%$ were enrolled in social health insurance.
} 
Table 1 Descriptive statistics of the study samples. EPBs Eerste polikliniek bezoeken (first hospital outpatient visits)

\begin{tabular}{|c|c|c|c|c|c|c|c|c|}
\hline & \multicolumn{4}{|c|}{ Orthopaedic care } & \multicolumn{4}{|c|}{ Neurosurgery } \\
\hline & Mean & SD & Minimum & Maximum & Mean & SD & Minimum & Maximum \\
\hline Bypassed nearest hospital & 0.38 & 0.49 & 0 & 1 & 0.54 & 0.50 & 0 & 1 \\
\hline Minimum extra travel time (min) & 6.77 & 5.90 & 0 & 54 & 5.76 & 5.43 & 0 & 51 \\
\hline \multicolumn{9}{|l|}{ Nearest hospital attributes } \\
\hline University medical centre & 0.07 & 0.26 & 0 & 1 & 0.09 & 0.29 & 0 & 1 \\
\hline Tertiary teaching hospital & 0.22 & 0.41 & 0 & 1 & 0.32 & 0.46 & 0 & 1 \\
\hline Hospital beds $(100 \times)$ & 5.42 & 2.28 & 1 & 14 & 6.14 & 2.03 & 2 & 14 \\
\hline Relevant EPBs $(100 \times)$ & 62.27 & 22.65 & 4 & 227 & 5.87 & 4.08 & 0 & 24 \\
\hline Waiting time below average & 0.49 & 0.50 & 0 & 1 & 0.43 & 0.50 & 0 & 1 \\
\hline \multicolumn{9}{|l|}{ Patient attributes } \\
\hline Female & 0.63 & 0.48 & 0 & 1 & 0.60 & 0.49 & 0 & 1 \\
\hline Age (years) & 53.65 & 18.13 & 18 & 90 & 52.56 & 15.13 & 18 & 90 \\
\hline Unemployed & 0.02 & 0.14 & 0 & 1 & 0.02 & 0.14 & 0 & 1 \\
\hline Incapacitated for work & 0.17 & 0.37 & 0 & 1 & 0.25 & 0.43 & 0 & 1 \\
\hline Retired & 0.35 & 0.48 & 0 & 1 & 0.26 & 0.44 & 0 & 1 \\
\hline On social security & 0.04 & 0.21 & 0 & 1 & 0.06 & 0.24 & 0 & 1 \\
\hline Self-employed & 0.02 & 0.14 & 0 & 1 & 0.02 & 0.14 & 0 & 1 \\
\hline Total EPBs in 2003 & 1.18 & 0.45 & 1 & 5 & 1.25 & 0.50 & 1 & 4 \\
\hline Urbanisation & 2.38 & 1.24 & 1 & 5 & 2.15 & 1.18 & 1 & 5 \\
\hline
\end{tabular}

unobserved individual characteristics. Patients older than 90 years were also excluded from our sample, because the (medical) condition of such patients is most often highly specific. We also omitted all observations on patients who travelled more than $60 \mathrm{~min}$, because it is likely that these patients were away from home when they needed hospital care. The resulting study sample contained 53,307 EPBs for orthopaedic care and 5,168 EPBs for neurosurgical care. Table 1 reports the descriptive statistics of all variables included in our specification.

\section{Dependent variable}

The dependent variable was simply assigned the value 1 when patient $i$ bypassed the nearest hospital and value 0 otherwise. ${ }^{3}$ Despite the fact that, in 2003, Dutch patients did not face any financial incentives to bypass the nearest hospital, numerous patients travelled to an alternative hospital. On average, patients in our sample travelled 15.7 min for an orthopaedic EPB and 18.4 min for a neurosurgical EPB. ${ }^{4}$ Because average travel time to the nearest hospital providing

\footnotetext{
${ }^{3}$ To test the robustness of our results, we also tried an alternative definition of the dependent variable; i.e. assigning value 1 only when patients bypassed the nearest hospital by travelling at least 5 min extra. This did not significantly alter the estimated coefficients.

${ }^{4}$ In this study estimated travel times refer to the fastest route by car. They are obtained by using a database that includes all fourdigit zip codes in the Netherlands and accounts for differences in average speed that exist between different road types.
}

orthopaedic care and neurosurgery is only 11.9 and $12.6 \mathrm{~min}$, respectively, these figures show that for both medical specialties a substantial number of patients went to a more distant hospital. For orthopaedic services almost four out of every ten patients did not visit the nearest hospital. The percentage of patients who bypassed the nearest hospital is even higher for neurosurgical services. Over $50 \%$ visited a more distant hospital than strictly necessary. ${ }^{5}$ For those patients who decided to bypass the nearest hospital, travel time on average increased by $10.0 \mathrm{~min}$ in the case of an orthopaedic EPB and by $10.8 \mathrm{~min}$ in the case of a neurosurgical EPB.

\section{Independent variables}

We expect the decision to bypass the nearest hospital to be negatively affected by travel time to the nextnearest hospital. Therefore, we calculated the extra time that is minimally required to reach another hospital in the case that a patient would decide to bypass the nearest one. Because hospital output quality was not measured in 2003, our specification includes several attributes of the nearest hospital as a proxy for its (perceived) quality: type of hospital, hospital size, and waiting time performance. Type of hospital is captured by two dummy variables. First, we constructed a variable that has value 1 when the nearest hospital is a

\footnotetext{
${ }^{5}$ Note that, in contrast to White and Morrisey [16], we explicitly control for individual hospitals' service offering. Orthopaedic care is provided by all Dutch hospitals, whereas neurosurgical services are offered by around two-thirds of the hospitals.
} 
university medical centre, and value 0 otherwise. Second, we constructed a variable that has value 1 when the nearest hospital is a tertiary medical teaching hospital and 0 otherwise. Data on hospital size was obtained from the Dutch Ministry of Health, Welfare and Sports. To capture possible care-specific size effects, we included not only the nearest hospital's number of beds but also its annual number of EPBs for orthopaedic care and neurosurgery, respectively. An issue that may arise in estimation of our logit model is whether it is smaller hospital size that increases the likelihood of hospital bypassing or higher bypass rates that lead to smaller hospital size. This possible endogeneity may bias the estimated coefficient for hospital size. In this paper, however, the latter variable is treated as exogenous. The fact that our model is static and does not deal with dynamic issues supports this assumption. Since it takes some time to adjust hospital capacity, the possible impact of patients' decisions to bypass the nearest hospital is a function of the cumulative number of patients over past years.

Data on individual hospital waiting times was obtained from the Netherlands Hospital Association. Since it was not compulsory for Dutch hospitals to report waiting times, this data contained many missing values. We therefore had to construct a dummy variable to test whether patients' hospital choice was affected by differences in waiting times. This variable has value 1 when the nearest hospital's average waiting time for an orthopaedic or neurosurgical EPB was known to be below the national average in 2003, and 0 otherwise. One could argue that hospital waiting time is affected by patients' bypassing decisions and is therefore endogenous, which may bias the estimated coefficient of hospital waiting time. Since this paper uses a static specification of hospital bypassing, however, we are able to treat hospital waiting time as exogenous.

Data on patient attributes was obtained from the Agis Health Database. From this database we were able to specify several socio-economic explanatory variables for each patient. The patient's age is included as a continuous variable, whereas gender is captured by a dummy variable that was assigned the value 1 for female patients. Each patient's total number of EPBs in 2003 and social status were also incorporated. ${ }^{6}$ The latter is captured by five different dummy variables that were assigned the value 1 when the patient was retired, unemployed, incapacitated for work, on social security, or self-employed, and the value 0 otherwise.

\footnotetext{
$\overline{6}$ Patients' own previous experiences with hospitals may also affect their bypass decisions. Unfortunately, such information is lacking in the database.
}

Using data from Statistics Netherlands we also specified a multinomial discrete variable to test for the possible effect of urbanisation. This variable was assigned the value 1 (very urban areas), 2 (urban areas), 3 (moderate urban areas), 4 (rural areas), or 5 (very rural areas). Although one might expect the opposite, the correlation matrix reveals that this explanatory variable is not highly correlated with the variable that captures minimum extra travel time (see Table 2).

\section{Estimation results}

\section{Orthopaedic care}

Our findings for orthopaedic care confirm the expected negative relationship between extra travel time required to reach the next-nearest hospital and the decision to bypass the nearest hospital (see Table 3 ). Holding all patient and hospital attributes constant, the results suggest that patients are more than $10 \%$ less likely to bypass their nearest hospital if going to an alternative hospital implies at least 5 min of extra travel time.

The patient attributes gender, unemployment and social security did not significantly affect the decision to bypass or use the nearest hospital for orthopaedic services. The likelihood of bypassing the nearest hospital decreases with patient age. As expected, older patients are less likely to bypass the nearest hospital. When patients retire, however, the probability that they visit a farther hospital increases. This suggests that the opportunity costs of increased travel time are lower for these patients. The same seems to hold for patients who are incapacitated to work. Self-employed people are also more likely to bypass the nearest hospital, as are patients who are admitted to a hospital more frequently and patients who live in rural areas. The latter result conflicts with our ex ante expectation. Apparently patients in rural areas are less averse to travel for orthopaedic care than patients in urban areas. This can perhaps be explained by the fact that those patients are already more used to travel for specific services like specialised health care, since these services are often not available locally.

We were surprised to find that, holding all other attributes constant, patients were almost $35 \%$ more likely to bypass the nearest hospital when this hospital was a university medical centre. ${ }^{7}$ The marginal effect for

\footnotetext{
$\overline{7}$ This result is not a spurious finding due to collinearity. Although there is some correlation between the explanatory variables university medical centre and number of beds (Table 2), exclusion of the latter does not change the sign and significance of the estimated coefficient.
} 
Table 2 Correlation matrix

\begin{tabular}{|c|c|c|c|c|c|c|c|}
\hline $\begin{array}{l}\text { Independent } \\
\text { variable }\end{array}$ & 1 & 2 & 3 & 4 & 5 & 6 & 7 \\
\hline 1 Urbanisation & & 0.18 & -0.07 & -0.20 & -0.16 & -0.01 & 0.0 \\
\hline $\begin{array}{l}2 \text { Minimum extra } \\
\text { travel time }\end{array}$ & 0.16 & & $-\mathbf{0 . 1 7}$ & -0.23 & -0.06 & -0.14 & -0.15 \\
\hline $\begin{array}{l}3 \text { University } \\
\text { medical centre }\end{array}$ & -0.03 & -0.18 & & $-\mathbf{0 . 1 5}$ & 0.06 & 0.43 & 0.0 \\
\hline $\begin{array}{l}4 \text { Tertiary } \\
\text { teaching } \\
\text { hospital }\end{array}$ & -0.32 & -0.04 & -0.22 & & 0.25 & 0.07 & 0.01 \\
\hline 5 Relevant EPBs & -0.09 & -0.23 & 0.68 & -0.01 & & 0.41 & -0.45 \\
\hline 6 Hospital beds & 0.18 & 0.11 & 0.46 & -0.11 & 0.43 & & -0.19 \\
\hline $\begin{array}{l}7 \text { Waiting time } \\
\text { below average }\end{array}$ & 0.25 & 0.11 & -0.22 & -0.53 & -0.10 & 0.07 & \\
\hline
\end{tabular}

Correlation coefficients for orthopaedic care are in bold. Correlation coefficients for neurosurgery are in italic

tertiary teaching hospitals is much smaller, but still positive and significant. The probability of a bypass increases by almost $6 \%$ when the nearest hospital is a tertiary hospital. In our opinion there are three plausible explanations for these results. First, GPs may advise patients to bypass these hospitals for their first outpatient visit. Research by the weekly magazine Elsevier in 2003 revealed that Dutch physicians, nurses, and hospital managers did not classify university medical centres among the best hospitals they know, despite their excellent medical expertise. It appeared that, according to the respondents, university hospitals especially suffered from bureaucracy [5]. Second, patients themselves may prefer admittance to a general hospital for their first hospital visit because of (perceived) quality differences that are particularly relevant to them, such as doctor communication skills and hospital staffs' responsiveness [12]. They may, for example, expect to get more personal attention in a general hospital than in a relatively large university medical centre that is aimed at scientific research. Furthermore, in the latter type of hospital it is far more likely for patients to be (initially) treated by a medical resident instead of a fully qualified physician. Third, and additional to the preceding demand side considerations, both university and tertiary teaching hospitals may be reluctant to accept too many patients for their first outpatient visit because they are oriented primarily towards providing highly specific care.

Patients, however, seem to prefer larger general hospitals over smaller ones. Hospital size, measured by the number of beds and the annual number of orthopaedic EPBs, significantly affects patients bypass decisions. Although the estimated marginal effects are rather small, on average patients are less likely to bypass the nearest hospital when it has more beds or treats more patients.
As expected, patients are significantly less likely to bypass their nearest hospital when they know its waiting time for an orthopaedic EPB is relatively low. The marginal effect of this hospital attribute, however, is quite small. A good waiting time performance of the nearest hospital decreases the probability of a bypass by only around $2 \%$.

\section{Neurosurgery}

For neurosurgery, the estimated marginal effects also reveal that patients are less likely to bypass the nearest hospital when travel time to the next-nearest hospital increases (see Table 3). A minimum extra travel time of $5 \mathrm{~min}$ decreases the probability of a bypass by approximately $6.5 \%$. Patient gender does not significantly affect hospital bypass decisions. The same holds for social security and self-employment. Holding all other attributes constant, older patients are less likely to travel farther than necessary for neurosurgical hospital care. That is, the likelihood of a bypass decision decreases with age. The opposite is true, however, once patients retire. After retirement the probability of bypassing the nearest hospital increases by almost $5 \%$. Unemployed patients in need of neurosurgical hospital care are also more likely to bypass the nearest hospital. Patients who are incapacitated to work are also more likely to bypass. Urbanisation has a significant and negative effect on patients' decisions not to visit the nearest hospital. Patients living in more rural areas are less likely to bypass the nearest hospital providing neurosurgery. This is not surprising because travel time is already relatively high for these patients as neurosurgical services are only available in larger (regional) hospitals. The total number of hospital admissions in a year, measured by EPBs per patient, does not significantly affect patients' bypass decisions for neurosurgical care. Patients who visited a hospital more frequently in 2003 are as likely to bypass the nearest hospital as patients who are referred to a hospital only once.

Again, the likelihood that a particular patient bypasses the nearest hospital strongly increases when this hospital is a university medical centre or a tertiary medical teaching hospital. ${ }^{8}$ These hospital attributes have positive marginal effects of almost 30 and $10 \%$, respectively. As mentioned before, we are not sure whether this effect reflects GPs' advices to patients, the

\footnotetext{
${ }^{8}$ Again, this result is not a spurious finding due to collinearity. Although there is some correlation between university medical centre and hospital size (Table 2), exclusion of the number of beds or neurosurgical EPBs does not change the sign and significance of the estimated coefficient.
} 
Table 3 Estimation results. ${ }^{\text {a }}$ Estimated standard errors (SE) are in parentheses

\begin{tabular}{|c|c|c|c|c|}
\hline & \multicolumn{2}{|l|}{ Orthopaedic care } & \multicolumn{2}{|l|}{ Neurosurgery } \\
\hline & Coefficients $(\mathrm{SE})^{\mathrm{a}}$ & Marginal effect & Coefficients (SE) & Marginal effect \\
\hline Minimum extra travel time (min) & $\begin{array}{l}-0.111 * * * \\
(0.002)\end{array}$ & $-2.27 \%$ & $\begin{array}{l}-0.065^{* * * *} \\
(0.008)\end{array}$ & $-1.33 \%$ \\
\hline \multicolumn{5}{|l|}{ Nearest hospital attributes } \\
\hline University medical centre & $\begin{array}{l}1.592 * * * \\
(0.048)\end{array}$ & $34.21 \%$ & $\begin{array}{l}1.785^{* * *} * \\
(0.214)\end{array}$ & $29.81 \%$ \\
\hline Tertiary teaching hospital & $\begin{array}{l}0.274^{* * * *} \\
(0.026)\end{array}$ & $5.70 \%$ & $\begin{array}{l}0.486^{* * * *} \\
(0.106)\end{array}$ & $10.02 \%$ \\
\hline Hospital beds $(100 \times)$ & $\begin{array}{l}-0.098^{* * * *} \\
(0.007)\end{array}$ & $-2.01 \%$ & $\begin{array}{l}-0.218^{* * * *} \\
(0.025)\end{array}$ & $-4.45 \%$ \\
\hline Relevant EPBs $(100 \times)$ & $\begin{array}{l}-0.004 * * * \\
(0.001)\end{array}$ & $-0.08 \%$ & $\begin{array}{l}-0.104 * * * \\
(0.013)\end{array}$ & $-2.13 \%$ \\
\hline Waiting time below average & $\begin{array}{l}-0.107 * * * \\
(0.024)\end{array}$ & $-2.19 \%$ & $\begin{array}{l}-0.490^{* * * *} \\
(0.096)\end{array}$ & $-10.39 \%$ \\
\hline \multicolumn{5}{|l|}{ Patient attributes } \\
\hline Female & $\begin{array}{c}0.009 \\
(0.021)\end{array}$ & $0.18 \%$ & $\begin{array}{l}-0.092 \\
(0.067)\end{array}$ & $-1.88 \%$ \\
\hline Age (years) & $\begin{array}{l}-0.013^{* * *} \\
(0.001)\end{array}$ & $-0.26 \%$ & $\begin{array}{l}-0.012^{* * *} \\
(0.004)\end{array}$ & $-0.24 \%$ \\
\hline Unemployed & $\begin{array}{c}0.047 \\
(0.075)\end{array}$ & $0.97 \%$ & $\begin{array}{l}0.671 \text { *** } \\
(0.251)\end{array}$ & $13.03 \%$ \\
\hline Incapacitated for work & $\begin{array}{l}0.207 * * * \\
(0.030)\end{array}$ & $4.31 \%$ & $\begin{array}{l}0.303^{* * * *} \\
(0.087)\end{array}$ & $6.14 \%$ \\
\hline Retired & $\begin{array}{l}0.152^{* * * *} \\
(0.041)\end{array}$ & $3.13 \%$ & $\begin{array}{c}0.229^{*} \\
(0.133)\end{array}$ & $4.63 \%$ \\
\hline On social security & $\begin{array}{c}-0.021 \\
(0.051)\end{array}$ & $-0.43 \%$ & $\begin{array}{l}-0.191 \\
(0.145)\end{array}$ & $-3.92 \%$ \\
\hline Self-employed & $\begin{array}{l}0.163^{* *} \\
(0.072)\end{array}$ & $3.39 \%$ & $\begin{array}{c}0.320 \\
(0.228)\end{array}$ & $6.41 \%$ \\
\hline Total EPBs in 2003 & $\begin{array}{l}0.250^{* * * *} \\
(0.022)\end{array}$ & $5.14 \%$ & $\begin{array}{c}0.102 \\
(0.068)\end{array}$ & $2.08 \%$ \\
\hline Urbanisation & $\begin{array}{l}0.143 * * * \\
(0.011)\end{array}$ & $2.73 \%$ & $\begin{array}{l}-0.123 * * * \\
(0.036)\end{array}$ & $-2.48 \%$ \\
\hline Constant & $\begin{array}{l}0.731 \text { *** } \\
(0.072)\end{array}$ & & $\begin{array}{l}2.916^{\text {**** }} \\
(0.248)\end{array}$ & \\
\hline Included observations & 48,778 & & 4,545 & \\
\hline Correct predictions & & & & \\
\hline Bypass $=0$ & $87 \%$ & & $53 \%$ & \\
\hline Bypass $=1$ & $33 \%$ & & $79 \%$ & \\
\hline Overall & $67 \%$ & & $68 \%$ & \\
\hline
\end{tabular}

$* * *$ Significance at $1 \%$, **significance at $5 \%$, *significance at $10 \%$

a To account for unobserved geographic differences we also included dummy variables capturing patients' province of residence. These estimation coefficients are available on request. Exclusion of these provincial dummy variables did not significantly alter the estimation results. Correct predictions are obtained when the predicted probability is $\leq 50 \%$ and the observed bypass $=0$, or when the predicted probability is $>50 \%$ and the observed bypass $=1$

latter's own preferences based on perceived quality differences, or admission restrictions imposed by these types of hospital. Just as we found for the orthopaedic sample, on average, patients prefer larger general hospitals above smaller ones for neurosurgical services. They are less likely to bypass the nearest hospital that provides these services when it has more beds and more neurosurgical EPBs.

For our neurosurgery sample we find a strong negative relationship between hospital waiting time performance and the likelihood of hospital bypassing.
Holding other attributes constant, patients were more than $10 \%$ less likely to bypass the nearest hospital that provides neurosurgery when its waiting time was known to be relatively low.

Differences between orthopaedic care and neurosurgery

Table 3 reveals similarities as well as differences regarding the factors affecting patients' hospital bypass decisions for orthopaedic care and neurosurgery. The 
first interesting difference between the two medical specialties analysed in this paper refers to patients' attitudes towards extra travel time. Although for both samples patients are less likely to bypass the nearest hospital when travel time to the next-nearest hospital increases, this effect is much stronger for orthopaedic EPBs than for neurosurgical EPBs. This result suggests that, in the case of more complex treatments, patients place a lower negative value on extra travel time, which is consistent with previous findings [16]. Another interesting difference concerns the estimated marginal effect for urbanisation. Whereas we find that patients from rural areas are more likely to bypass the nearest hospital for orthopaedic care, we find the opposite for neurosurgery. Our explanation for this result is that in rural areas a substantial number of patients are not able to visit the hospital closest to their home for neurosurgical care because it simply does not offer such services. These patients may therefore be less likely to bypass the nearest hospital providing the care they need than patients in the orthopaedic sample, as for the latter admission to the geographically closest hospital is always feasible. The third difference that catches the eye is perhaps the most interesting. Hospital waiting time performance appears to have a much stronger effect on patients' bypass decisions for neurosurgical services than for orthopaedic services. Apparently, the valuation of shorter waiting time varies with types of hospital care. The importance of waiting time as a determinant of hospital bypass decisions seems to be more important for complex procedures.

\section{Conclusion}

Despite the absence of financial incentives, in 2003 numerous Dutch patients bypassed the nearest hospital for both orthopaedic care $(38 \%)$ and neurosurgery (54\%). The estimation results of our logit specification reveal that extra travel time and hospital waiting time performance significantly affect the decisions made by patients to visit or bypass the hospital closest to their homes. As expected, we find a negative relationship between extra travel time and hospital bypassing. Relatively low waiting time also significantly decreases the likelihood of patients deciding to bypass the nearest hospital. Patients, however, seem to place a lower negative value on extra travel time for orthopaedic care than for neurosurgery. The valuation of shorter waiting time also varies between these two types of hospital care. A good performance of the nearest hospital on waiting time decreases the likelihood of a bypass most for neurosurgery. We are surprised to find that, in both samples, patients were more likely to bypass the nearest hospital when this was a university medical centre or a tertiary teaching hospital. Apparently patients did not prefer admission to such hospitals for their initial visit. In addition to travel time and hospital attributes, patient attributes, such as age and social status, also significantly affected hospital bypass decisions. These results have important policy implications for European health planners, hospitals, and especially health insurers who are marketing health plans with a limited set of providers. To properly assess the substitutability of hospitals that underlies hospital market power, they explicitly have to take both patient and hospital care heterogeneity into account.

Acknowledgments The authors thank Erik Schut, Karin Monstad, Wynand van de Ven, René van Vliet, and two anonymous reviewers for helpful comments. They also thank Agis for provision of data. Draft versions of this paper were presented at the 5th World Congress of the International Health Economics Association in Barcelona (10-13 July 2005) and the 6th European Conference on Health Economics in Budapest (6-9 July 2006).

\section{References}

1. Bronstein, J.M., Morrisey, M.A.: Bypassing rural hospitals for obstetrics care. J. Health Polit. Policy Law 16, 87-118 (1991)

2. Calem, P.S., Rizzo, J.A.: Competition and specialization in the hospital industry: an application of Hotelling's location model. South. Econ. J. 61, 1182-1198 (1995)

3. Cutler, D.M.: Equality, efficiency, and market fundamentals: the dynamics of international medical care reform. J. Econ. Lit. 40, 881-906 (2002)

4. ECORYS-NEI: Vraagfactoren ziekenhuizen (Determinants of Hospital Choice), ECORYS-NEI, Rotterdam (2003)

5. Elsevier: De beste ziekenhuizen (The Best Hospitals), 27 September, pp. 38-50. Elsevier, The Netherlands (2003)

6. Lee, H.L., Cohen, M.A.: A multinominal logit model for the spatial distribution of hospital utilization. J. Bus. Econ. Stat. 3, 159-168 (1985)

7. Maynard, A.: European health policy challenges. Health Econ. 14, S255-S263 (2005)

8. Montefiori, M.: Spatial competition for quality in the market for hospital care. Eur. J. Health Econ. 6, 131-135 (2005)

9. NMa: Besluit inzake de voorgenomen fusie tussen Ziekenhuis Hilversum en Ziekenhuis Gooi-Noord (Decision concerning the proposed merger between the hospitals Hilversum and Gooi-Noord). Netherlands Competition Authority (2005)

10. Van der Schee, E., Delnoij, D., Kerssens, J.: Keuze van ziekenhuizen: welke overwegingen zijn belangrijk voor consumenten? (Hospital choice: which considerations are important for consumers?). Tijdschr. Geneeskd. 83, 113-115 (2005)

11. Schut, F.T., van de Ven, W.P.M.M.: Rationing and competition in the Dutch health-care system. Health Econ. 14, S59 S74 (2005) 
12. Sofaer, S., Crofton, C., Goldstein, E., Hoy, E., Crabb, J.: What do consumers want to know about the quality of care in hospitals? Health Serv. Res. 40, 2018-2036 (2005)

13. Sorensen, A.T.: Insurer-hospital bargaining: negotiated discounts in post-deregulated Connecticut. J. Ind. Econ. 51, 469-490 (2003)

14. Tai, W.C., Porell, F.W., Adams, E.K.: Hospital choice of rural Medicare beneficiaries: patient, hospital attributes, and the patient-physician relationship. Health Serv. Res. 39, 1903-1922 (2004)

15. Xavier, A.: Hospital competition, GP fundholders and waiting times in the UK internal market: the case of elective surgery. Int. J. Health Care Finance Econ. 3, 25-51 (2003)

16. White, W.D., Morrisey, M.A.: Are patients travelling further?. Int. J. Econ. Bus. 5, 203-221 (1998) 